TITLE:

\title{
New consideration on flutter properties based on step-by-step analysis
}

\section{$\operatorname{AUTHOR}(\mathrm{S})$ :}

Matsumoto, Masaru; Matsumiya, Hisato; Fujiwara, Shinya; Ito, Yasuaki

\section{CITATION:}

Matsumoto, Masaru ...[et al]. New consideration on flutter properties based on step-bystep analysis. Journal of Wind Engineering and Industrial Aerodynamics 2010, 98(8-9): 429-437

ISSUE DATE:

2010-08

URL:

http://hdl.handle.net/2433/130705

\section{RIGHT:}

(C) 2010 Elsevier Ltd; This is not the published version. Please cite only the published version.; この論文は出版社版でありません。引用の際に は出版社版をご確認ご利用ください。 


\section{Title:}

New Consideration on Flutter Properties based on Step-by-Step Analysis

\section{Authors:}

Masaru Matsumoto 1)

Hisato Matsumiya 2)

Shinya Fujiwara 3)

Yasuaki Ito 4)

\section{$\underline{\text { Affiliations: }}$}

1) Professor emeritus at Kyoto University, Japan

2) Central Research Institute of Electric Power Industry, Japan

3) OSAKA GAS CO. LTD., Japan

4) Shimizu Corporation, Japan

\section{Corresponding Author:}

Masaru Matsumoto

Professor emeritus at Kyoto University, Japan

TEL: $\quad 81-774-32-5931$

FAX: $\quad 81-774-32-5931$

E-mail: matsu@brdgeng.gee.kyoto-u.ac.jp 


\begin{abstract}
This paper studies the coupled flutter mechanism of plate and long span bridges based on Step-by-step analysis (SBS). Fundamental flutter modes are defined based on amplitude ratio and phase difference between heaving and torsional motions. Furthermore, a formula remarkably similar to the Selberg formula can be derived by use of the particular simplified flutter-onset condition. In the process of SBS analysis, some torsional divergent velocities where the torsional rigidity becomes zero can be defined. Finally, the flutter-behavior of an elastic model of the complete Akashi-Kaikyo Bridge, which is the longest suspension bridge in the world, is studied from the point of view of flutter in two-degrees of freedom, namely heaving and torsional motion, taking into account the structural coupling effect of additional torsional displacement induced by horizontal displacement as a structural coupling property.
\end{abstract}

\title{
Keywords:
}

Step-by-Step Analysis, Fundamental Flutter Mode, Selberg's Formula, Torsional Divergence Velocity, 2DOF Coupling Flutter affected by Structural Coupling 


\section{Introduction}

The authors reported that flutter characteristics, including velocity-frequency characteristics $V$ - $\omega$, velocity-damping characteristics $V$ - $\delta$, velocity-amplitude ratio characteristics $V$ - $\eta_{0} / \phi_{0}$, and velocity-phase difference characteristics $V$ - $\Psi$, obtained by step-by-step analysis (SBSA) (Matsumoto et al, 1995) and Complex-Eigen-Value analysis (CEVA) showed perfect agreement to within 6 digits (Matsumoto et al, 2007). In this paper, the physical meanings of coupled flutter branch of thin plates and plate-like bodies are discussed in relation to fundamental flutter modes by using SBSA. Also, the flutter onset velocity and branch switch characteristics are investigated based on these two fundamental flutter modes. Furthermore, it is clarified that torsional divergence is classified into static $1 \mathrm{DOF}$ torsional divergence and dynamic 2 degree of freedom (DOF) torsional divergence. Moreover, an equation similar in form to Selberg's formula (Selberg, 1961), which evaluates flutter onset velocity, can be obtained based on SBSA of torsional branch (TB) characteristics. Besides, in this study focusing on the horizontal displacement and sequential torsional displacement as structural properties, 2DOF aerodynamic coupling flutter instability affected by structural coupling between horizontal and torsional displacements have been considered in flutter analysis instead of 3DOF modes, those are the vertical bending mode, torsional mode and horizontal bending mode of long span suspension bridges with truss-stiffened girder.

\section{STEP-BY-STEP FLUTTER ANALYSIS}


By using the aerodynamic derivatives, $H_{\mathrm{i}}{ }^{*}$ and $A_{\mathrm{i}}{ }^{*}$ (i=1 4), proposed by Scanlan, the heaving and torsional 2DOF differential equations are expressed as follows;

$$
\begin{aligned}
& m \ddot{\eta}+c_{\eta 0} \dot{\eta}+k_{\eta 0} \eta=\rho b^{2} \omega_{F} H_{1}^{*} \dot{\eta}+\rho b^{2}{\omega_{F}}^{2} H_{4}^{*} \eta+\rho b^{3} \omega_{F} H_{2}^{*} \dot{\phi}+\rho b^{3} \omega_{F}{ }^{2} H_{3}^{*} \phi \\
& I \ddot{\phi}+c_{\phi 0} \dot{\phi}+k_{\phi 0} \phi=\rho b^{3} \omega_{F} A_{1}^{*} \dot{\eta}+\rho b^{3} \omega_{F}{ }^{2} A_{4}^{*} \eta+\rho b^{4} \omega_{F} A_{2}^{*} \dot{\phi}+\rho b^{4} \omega_{F}{ }^{2} A_{3}^{*} \phi
\end{aligned}
$$

where $\eta$ and $\phi$ are the heaving and torsional displacements, $m$ and $I$ are the mass and mass inertia per unit length, $c_{\eta 0}$ and $c_{\phi 0}$ are the damping coefficients of heaving and torsional vibrations, $k_{\eta 0}$ and $k_{\phi 0}$ are the heaving and torsional stiffness, $\rho$ is the air density, $b$ is the half chord length, $\omega_{F}$ is the circular frequency, $k$ is the reduced frequency $\left(=b \omega_{F} / V\right)$, and $V$ is the wind velocity.

In solving the $2 \mathrm{DOF}$ flutter equations, the commonly used method is the Complex Eigen-Value analysis (CEVA). In CEVA, the four flutter characteristics are solved based on an eigen-value problem. On the other hand, in step-by-step analysis (SBSA), reported by the authors (Matsumoto et al, 1995), in which the flutter frequency in the heaving branch $(\mathrm{HB})$ and $\mathrm{TB}$ are converged by iterative calculation, there are some discrepancies in flutter values velocity-frequency characteristics $V$ - $\omega$, velocity-damping characteristics $V$ - $\delta$, velocity-amplitude ratio characteristics $V$ - $\eta_{0} / \phi_{0}$, and velocity-phase difference characteristics $V-\Psi$, between numerical results obtained by CEVA and SBSA at higher reduced velocity than the flutter onset reduced velocity. Therefore, both flutter frequency and damping have been simultaneously converged in iteration calculation in order to resolve the difference in flutter values in the two different analyses (Matsumoto et al, 2007). This modified SBSA is applied in flutter analysis in the following series of analyses described below. The brief analytical process in TB is as follows; 
Step 1) In a torsional system, torsional motion is assumed taking the damping in consideration.

$$
\phi=\phi_{0} e^{-\zeta_{F}^{\prime} \omega_{F} t} \sin \omega_{F} t
$$

where $\phi_{0}$ is the amplitude of torsional motion, $\zeta_{F}^{\prime}=\zeta_{F} / \sqrt{1-\zeta_{F}{ }^{2}}, \zeta_{F}$ is the damping ratio, and $t$ is the time.

Step 2) Heaving motion is generated by torsional motion as forced vibration, with a certain amplitude ratio and phase difference.

$$
\ddot{\eta}+2 \zeta_{\eta}^{*} \omega_{\eta}^{*} \dot{\eta}+\omega_{\eta}^{* 2} \eta=\left(\frac{\rho b^{3}}{m}\right) \omega_{F} H_{2}^{*} \dot{\phi}+\left(\frac{\rho b^{3}}{m}\right) \omega_{F}^{2} H_{3}^{*} \phi
$$

where,

$$
\zeta_{\eta}^{*}=\left(2 \zeta_{\eta 0} \omega_{\eta 0}-\frac{\rho b^{2}}{m} \omega_{F} H_{1}^{*}\right) / 2 \omega_{\eta}^{*}, \omega_{\eta}^{*}=\sqrt{\omega_{\eta 0}{ }^{2}-\frac{\rho b^{2}}{m} \omega_{F}{ }^{2} H_{4}^{*}}
$$

where $\zeta_{\eta 0}$ is the heaving natural damping ratio and $\omega_{\eta 0}$ is the heaving natural circular frequency.

Step 3) Torsional motion is also generated by heaving motion as free vibration. Finally, the flutter logarithmic damping $\left(\delta_{F}\right)$ and the flutter circular frequency $\left(\omega_{F}\right)$ are calculated with the following equations.

$$
\begin{aligned}
\delta_{F}= & 2 \zeta_{\phi 0} \omega_{\phi 0} \frac{\pi}{\omega_{F}}-\pi \Omega_{1} A_{2}^{*}-\pi \Omega_{1} \Omega_{2}\left\{A_{1}^{*}\left|H_{2}^{*}\right|\left(1+\zeta_{F}^{\prime 2}\right)^{1 / 2}\left(\zeta_{F}^{\prime} \sin \theta_{1}+\cos \theta_{1}\right)\right. \\
& \left.+A_{1}^{*}\left|H_{3}^{*}\right|\left(\zeta_{F}^{\prime} \sin \theta_{2}+\cos \theta_{2}\right)-A_{4}^{*}\left|H_{2}^{*}\right|\left(1+\zeta_{F}^{\prime 2}\right)^{1 / 2} \sin \theta_{1}-A_{4}^{*}\left|H_{3}^{*}\right| \sin \theta_{2}\right\} \\
\omega_{F}= & {\left[\left\{\omega_{\phi 0}{ }^{2}-\Omega_{1}{\omega_{F}}^{2} A_{3}^{*}-\Omega_{1} \Omega_{2}{\omega_{F}}^{2}\left\{A_{1}^{*}\left|H_{2}^{*}\right|\left(1+\zeta_{F}^{\prime 2}\right)^{3 / 2} \sin \theta_{1}\right.\right.\right.} \\
& +A_{1}^{*}\left|H_{3}^{*}\right|\left(1+\zeta_{F}^{\prime 2}\right) \sin \theta_{2}+A_{4}^{*}\left|H_{2}^{*}\right|\left(1+\zeta_{F}^{\prime 2}\right)^{1 / 2}\left(\cos \theta_{1}-\zeta_{F}^{\prime} \sin \theta_{1}\right) \\
& \left.\left.+A_{4}^{*}\left|H_{3}^{*}\right|\left(\cos \theta_{2}-\zeta_{F}^{\prime} \sin \theta_{2}\right)\right\}\left(1-\zeta_{F}{ }^{2}\right)\right]^{1 / 2}
\end{aligned}
$$

where $\zeta_{\phi 0}$ is the torsional natural damping ratio and $\omega_{\phi 0}$ is the torsional natural circular frequency and, 


$$
\Omega_{1}=\left(\frac{\rho b^{4}}{I}\right), \Omega_{2}=\left(\frac{\rho b^{2}}{m}\right) \omega_{F}^{2} /\left(\left(\zeta_{F}^{\prime 2} \omega_{F}^{2}-\omega_{F}^{2}-2 \zeta_{\eta}^{*} \zeta_{F}^{\prime} \omega_{\eta}^{*} \omega_{F}+\omega_{\eta}^{* 2}\right)^{2}+\left(2 \zeta_{\eta}^{*} \omega_{\eta}^{*} \omega_{F}-2 \zeta_{F}^{\prime} \omega_{F}^{2}\right)^{2}\right\}^{1 / 2}
$$

Step 4) Then, convergence calculation associated with the flutter frequency in Step 3 and the originally assumed flutter frequency in Step 1, is carried out.

Then, all of the flutter values obtained by this modified SBSA show completely identical values to those obtained by CEVA, exceptionally branch switch, up to six digits in analyzed values. Fig. 1 shows the comparison of flutter values for a rectangular cylinder with $B / D=20$ obtained by numerical analysis, by CEVA and SBSA, and wind tunnel tests result.

\section{Fundamental flutter mode}

\subsection{Fundamental flutter modes in torsional and heaving 2DOF coupled flutter}

Torsional fundamental mode is defined as substantially torsional vibration around a certain point apart from the mid-chord point. In this mode, the phase difference, between torsional (noses-up positive) and heaving response (downward positive) at the mid-chord point is $0^{\circ}$ or $180^{\circ}$, and the torsional twist center is a point upstream or downward from the mid-chord point, respectively. These fundamental modes are expressed by $\mathrm{T}_{0}$ or $\mathrm{T}_{180}$. As shown in Fig. 2(a), (b), in these modes, torsional response would be excited by the pitching moment $A_{1}{ }^{*} \dot{\eta}$ induced by the relative pitching angle by $\dot{\eta} / V$, in which $\eta$ is the heaving displacement at the mid-chord point.

On the other hand, the heaving fundamental mode is defined as a prominent heaving response induced by lift generated by a slight pitching angle in the quasi-steady sense with $-90^{\circ}$ or $90^{\circ}$ as the phase lag of heaving to torsional displacements. These two fundamental modes correspond to $\mathrm{d} C_{L} / \mathrm{d} \alpha>0$ or $\mathrm{d} C_{L} / \mathrm{d} \alpha<0$ and are expressed by $\mathrm{H}_{-90}$ or 
$\mathrm{H}_{90}$, respectively. In the heaving mode, the heaving response is excited by the lift force $H_{3}{ }^{*} \phi$ by a slight torsional response $\phi$ in the quasi-steady state. $\mathrm{H}_{-90}$ and $\mathrm{H}_{90}$ are illustrated in Fig. 2(c), (d).

By taking into account the fundamental mode definition, the flutter mode in coupled flutter can be resolved into two fundamental modes by using the phase difference $\Psi$, in which $\Psi$ is defined as the heaving lag to torsional response in Eq. (8). Also, the contribution of each fundamental mode is expressed by Eq. (9).

$$
\begin{aligned}
& \phi=\phi_{0} \sin \omega t, \quad \eta=\eta_{0} \sin (\omega t-\Psi) \\
& T_{180}=-\cos \Psi, H_{90}=\sin \Psi\left(90^{\circ} \leq \Psi \leq 180^{\circ}\right) \\
& T_{0}=\cos \Psi, H_{90}=\sin \Psi\left(0^{\circ} \leq \Psi \leq 90^{\circ}\right) \\
& T_{0}=\cos \Psi, H_{-90}=-\sin \Psi\left(-90^{\circ} \leq \Psi \leq 0^{\circ}\right) \\
& T_{180}=-\cos \Psi, H_{-90}=-\sin \Psi\left(-180^{\circ} \leq \Psi \leq-90^{\circ}\right)
\end{aligned}
$$

\subsection{Relation between flutter branch and fundamental flutter modes}

The flutter fundamental modes are closely related to the flutter branch of SBSA explained as follows.

\section{Torsional branch}

Step 1: Torsional oscillation.

$$
\phi(t)=\phi_{0} e^{i \omega_{\phi} t} e^{-\zeta_{\phi} \omega_{\phi} t}
$$

Step 2: Unsteady lifts, $H_{2}{ }^{*} \dot{\phi}$ and $H_{3}{ }^{*} \phi$, act on the $\eta$ system as forced-vibration forces. Then, the $\eta$ response with $\omega_{\phi}$ can be excited. In this step, the amplitude ratio and phase difference between heaving and torsional response can be characterized. 
Step 3: Unsteady moment, $A_{1}{ }^{*} \dot{\eta}$ and $A_{4}{ }^{*} \eta$, generated by the heaving response at step

2 , act on the torsional system as self excited moments, then the flutter frequency $\omega_{F}$ $\left(=\omega_{\phi}\right)$ and the flutter damping $\delta_{F}\left(=\delta_{\phi}\right)$ can be characterized, if $\delta_{\phi}$ and $\omega_{\phi}$ in Step 1 are identical to those in Step 3. In this, $H_{1}{ }^{*} \dot{\eta}$ is the self excited pitching moment induced by $\dot{\eta}$, therefore, the torsional response should correspond to the fundamental flutter modes $\mathrm{T}_{0}$ and $\mathrm{T}_{180}$.

\section{$\underline{\text { Heaving Branch }}$}

Step 1: Heaving oscillation.

$$
\eta(t)=\eta_{0} e^{i \omega_{\eta} t} e^{-\zeta_{\eta} \omega_{\eta} t}
$$

Step 2: Unsteady moments, $A_{1}{ }^{*} \dot{\eta}$ and $A_{4}{ }^{*} \eta$ act on the torsional system as forced vibration-forces. Then, the $\phi$ response with $\omega_{\eta}$ can be excited. In this step, the amplitude ratio and phase difference between heaving and torsional response can be characterized.

Step 3: Unsteady lift, $H_{2}{ }^{*} \dot{\phi}$ and $H_{3}{ }^{*} \phi$, generated by the torsional response at Step 2, act on the heaving system as self excited lift forces, then flutter frequency $\omega_{F}\left(=\omega_{\eta}\right)$ and flutter damping $\delta_{F}\left(=\delta_{\eta}\right)$ can be characterized, if $\delta_{\eta}$ and $\omega_{\eta}$ at Step 1 are identical to those at Step 3. In this, $H_{3}{ }^{*} \phi$ is the unsteady lift induced by the relative pitching angle due to the torsional response $\phi$, and the heaving response induced by self excited action of $\mathrm{H}_{3}{ }^{*} \phi$ should correspond the flutter fundamental modes $\mathrm{H}_{-90}$ and $\mathrm{H}_{90}$.

Thus it is verified that in $\mathrm{TB}, \mathrm{T}_{0}$ and $\mathrm{T}_{180}$ are classified as self-excite terms, and on the other hand $\mathrm{H}_{-90}$ and $\mathrm{H}_{90}$ are forced terms. By contrast, in $\mathrm{HB}, \mathrm{H}_{-90}$ and $\mathrm{H}_{90}$ are subjected to self-excited terms and $\mathrm{T}_{0}$ and $\mathrm{T}_{180}$ to forced terms. 


\subsection{Relations between flutter onset, branch switch and fundamental flutter modes}

By using Eq. (9) and the phase difference $\Psi$, the flutter modes are resolved as shown in Fig. 3. As shown, for the case of a frequency ratio of $f_{\phi 0} / f_{\eta 0}=1.3$, flutter onsets in TB at $V=9.6[\mathrm{~m} / \mathrm{s}]$ and the flutter major branch switches from TB to $\mathrm{HB}$ at $V=11.1[\mathrm{~m} / \mathrm{s}]$. Comparing these characteristics, velocities, and the flutter fundamental modes, it is clarified that for TB when the self excited term $\mathrm{T}_{0}(=\cos \Psi)$ becomes large, flutter might onset. Furthermore, when the self-excited-term $\mathrm{H}_{-90}(=-\sin \Psi)$ becomes large, branch switching seems to occur. However, there remains some questions in $\mathrm{TB}$, as the maximum value of $\mathrm{T}_{0}$ does not correspond to the flutter onset velocity. On the other hand, when $\mathrm{H}_{-90}$ becomes large enough in $\mathrm{HB}$, branch switching from $\mathrm{TB}$ to $\mathrm{HB}$ seems to occur. Therefore, more details should be studied, taking into account the flutter fundamental modes. These give us some hints about the physical generation mechanism and branch switching of coupled flutter.

\section{Formula similar to Selberg's formula to predict the flutter onset velocity of thin plate}

The following is a discussion about Selberg's formula (Selberg, 1961) which is a well-known evaluation formula for the flutter onset velocity for a thin plate, as described by Eq. (12).

$$
\left.V_{F}=3.71 \cdot f_{\phi 0} \cdot(2 b) \sqrt{\frac{\sqrt{m I}}{\rho(2 b)^{3}}\left\{1-\left(\frac{f_{\eta 0}}{f_{\phi 0}}\right)^{2}\right.}\right\}
$$

where $f_{\phi 0}$ is the torsional natural frequency and $f_{\eta 0}$ is the heaving natural frequency. 
Then, authors investigated the formula to predict the flutter onset velocity by using SBSA. It was clarified that the flutter onset velocity almost coincides with the crossing point of the $\omega_{\eta^{-}}-V$ diagram and the $\omega_{\phi^{-}} V$ diagram of TB, as shown in Fig. 4. Then, the following assumptions are used.

Assumption 1: Quasi-steady state; $F(k)=1$, and $G(k)=0$, where Theodorsen function $C(k)=F(k)-i G(k)$.

Assumption 2: Flutter occurs, at the crossing point of the $\omega_{\eta^{-}} V$ diagram and the $\omega_{\phi^{-}} V$ diagram.

Assumption 3: $\square \omega_{\eta}=\omega_{\eta 0}$ at any velocity, and $\omega_{\phi}$ in 2DOF can be approximately expressed by $\omega_{\phi}$ in $1 \mathrm{DOF}$.

The torsional 1DOF frequency is described by Eq. (13).

$$
\omega_{\phi}^{\prime}=\sqrt{\omega_{\phi 0}^{2}-\left(\frac{\rho b^{4}}{I}\right) \omega_{\phi}^{2} A_{3}^{*}}
$$

Then, aerodynamic derivatives are described by Eq. (14).

$$
A_{3}^{*}=\frac{\pi}{k}\left(\frac{F(k)}{k}+\frac{G(k)}{2}\right)=\frac{\pi}{k^{2}}=\frac{V^{2} \pi}{\left(b \omega_{\phi}\right)^{2}}
$$

Using the assumption of $F(k)=1, G(k)=0$ (Assumption 1), the torsional 1DOF frequency is described by Eq. (15).

$$
\omega_{\phi}^{*}=\sqrt{\omega_{\phi 0}^{2} /\left(1+\frac{\rho b^{4}}{I} \cdot \frac{\pi}{k^{2}}\right)}
$$

When flutter occurs, using $\omega_{F}=\omega_{\eta}=\omega_{\phi}$ (Assumption 2) and $\omega_{\eta}=\omega_{\eta 0}$ and $\omega_{\phi}$ (2DOF) $=\omega_{\phi}$ (1DOF) (Assumption 3), the following equation can be derived, where, $k=b \omega_{F} / V=b \omega_{\eta} / V=b \omega_{\eta 0} / V$ (because of TB). 


$$
V_{r}=f_{\phi 0} \sqrt{\frac{4 \pi I}{\rho b^{4}}\left\{1-\left(\frac{f_{\eta 0}}{f_{\phi 0}}\right)^{2}\right\}}
$$

Eq. (16) is transformed to the following equation.

$$
V_{F}=\sqrt{\frac{8 \pi \sqrt{I}}{b \sqrt{m}}} \cdot f_{\phi 0} \cdot 2 b \sqrt{\frac{\sqrt{m I}}{\rho(2 b)^{3}}\left\{1-\left(\frac{f_{\eta 0}}{f_{\phi 0}}\right)^{2}\right\}}
$$

Eq. (17) is similar to Selberg's formula as described by Eq. (12), and the coefficient of Eq. (17) is calculated as follows. Eq. (18) and Eq. (19), relationships between $m$ and $I$ in thin plate, can be obtained.

$$
\begin{aligned}
& I=\int_{A} \rho_{s} r^{2} d A=\int_{A} \rho_{s}\left(y^{2}+z^{2}\right) d A \\
& =\int_{y} \int_{z} \rho_{s}\left(y^{2}+z^{2}\right) d y d z=\rho_{s}\left(\frac{4 b d^{3}}{3}+\frac{4 b^{3} d}{3}\right) \\
& I=\lim _{d \rightarrow 0}\left(4 \rho_{s} b d\right)\left(\frac{1}{3} d^{2}+\frac{1}{3} b^{2}\right) \cong \frac{1}{3} m b^{2}
\end{aligned}
$$

where $d$ is the half of depth.

The relationships between $m$ and $I$ in a thin plate are described as $I=m b^{2} / 3$, and Eq. (17) can be modified to be like Selberg's Form as follows;

$$
V_{F}=3.81 \cdot f_{\phi 0} \cdot(2 b) \sqrt{\frac{\sqrt{m I}}{\rho(2 b)^{3}}\left\{1-\left(\frac{f_{\eta 0}}{f_{\phi 0}}\right)^{2}\right\}}
$$

As shown in Fig. 5, the flutter onset velocity of a thin plate obtained by CEVA or SBSA, Selberg's formula, and Eq. (20) are compared for various frequency ratios. From this figure, the flutter onset velocity obtained by Eq. (20) agrees fairly well with that obtained by SBSA as well as Selberg's formula. 


\section{Torsional divergence}

Torsional divergence could occur when the restoring force becomes smaller than the pitching moment force in the static state. On the other hand, for a dynamic system, when the torsional rigidity becomes zero due to aerodynamic unsteady pitching moment, divergence occurs. Therefore, by using SBSA, static divergence and dynamic torsional divergence can be easily studied. Torsional divergences are classified into the following three different types.

(a) $1 \mathrm{DOF}$ divergence: Torsional rigidity is zero at Step 1 of TB.

$$
\omega_{\phi}^{2}=\omega_{\phi 0}^{2}-\left(\rho b^{4} / I\right) \omega_{\phi}^{2} A_{3}^{*}=0
$$

In the quasi-steady state $\left(A_{3}{ }^{*}=2 C_{M}{ }^{\prime} / k^{2}\right)$, the critical velocity is identical to the static one as given by Eq. (20).

$$
V_{c r}=\sqrt{\frac{2 k_{\phi 0}}{\rho B^{2} C_{M}^{\prime}}}
$$

Where, $k$ is the reduced frequency $(k=b \omega / V), k_{\phi 0}$ is the structural torsional rigidity at $V=0[\mathrm{~m} / \mathrm{s}]$ and $C_{M}{ }^{\prime}$ is the slope of the pitching moment coefficient.

(b) 2DOF dynamic divergence: Torsional rigidity is zero at Step 3 of TB.

$$
\begin{aligned}
\omega_{F}^{2}= & \omega_{\phi 0}^{2}-\left(\rho b^{3} / I\right) \omega_{F}^{2}\left\{b A_{3}^{*}-A_{4}^{*}\left(\eta_{0} / \phi_{0}\right)\left(\cos \Psi-\zeta_{F} \sin \Psi\right)\right. \\
& \left.-A_{1}^{*}\left(\eta_{0} / \phi_{0}\right)\left(\zeta_{F}^{2}+1\right) \sin \Psi\right\}=0
\end{aligned}
$$

(c) 1 DOF dynamic divergence: Torsional rigidity is zero at Step 2 of HB.

$$
\omega_{\phi}^{* 2}=\omega_{\phi 0}^{2}-\left(\rho b^{4} / I\right) \omega_{F}^{2} A_{3}^{*}=0
$$


These three different divergence critical velocities of thin plate with a frequency ratio $f_{\phi 0} / f_{\eta 0}=1.3$ are indicated on a $V-f$ diagram as shown in Fig. 6 . The critical velocities of these phenomena might be affected by frequency ratios among $f_{\phi 0} / f_{\eta 0}$, but the details should be studied in a future study.

Measured 1DOF torsional divergence critical velocities of a rectangular cylinder with $B / D=20$ under three different test conditions are compared with calculated results from Eq. (22) in Table 1. Taking into account that velocity pitch is $0.5[\mathrm{~m} / \mathrm{s}]$ in this test, fairy good agreement can be seen.

On the other hand, 2DOF torsional divergence is observed in the test of torsional free-vibration under the heaving forced-vibration system.

\section{2DOF Coupled flutter affected by structural coupling of full elastic suspension bridge with truss-stiffened girder (Case of Akashi Strait Bridge)}

Recently coupled flutter instability has been analyzed with 3DOF, that is analysis with vertical displacement $\eta$ (heaving displacement), horizontal displacement $\xi$, and torsional displacement $\phi$, instead of conventional 2DOF $(\eta, \phi)$ analysis (Matsumoto et al, 1995). The experimental results on flutter characteristics of the Akashi Strait Bridge elastic scale model (AFM, 1/100 scale, 40m total span length) as shown in Photo 1 were the background to the 3DOF analysis. It has been reported that damping-velocity characteristics could not always be explained by conventional 2DOF flutter analysis (Miyata et al, 1994). 3DOF flutter analysis additionally taking account of horizontal motion and aerodynamic forces caused by horizontal motion of the bridge girder, can show a better fit to test results as shown in Fig. 7 (Sato et al, 1996). However, there are 
some questions why 3DOF flutter analysis can better fit the test results, even though the aerodynamic derivative associated with horizontal motion $\xi$ is much smaller than the other derivatives associated with heaving $\eta$, and torsional motion $\phi$ as shown in Fig. 8. Furthermore, looking at the video film of the flutter characteristics of the AFM, the flutter mode is significantly similar to those in the $2 \mathrm{DOF}$ system of a flat rectangular cylinder with $B / D=20$ ( $B$ : chord length, $D$ : depth). In consequence, flutter of the AFM could be conventional 2DOF coupled flutter, and the discrepancy of the flutter onset velocity between test results and analytical results could be caused by another reason. Therefore, in this study, focusing on the horizontal displacement and sequential torsional displacement, 2DOF aerodynamic coupling and structural coupling between horizontal and torsional displacements have been considered in flutter analysis.

By using the aerodynamic derivatives, $H_{\mathrm{i}}{ }^{*}, A_{\mathrm{i}}{ }^{*}$ and $P_{\mathrm{i}}{ }^{*}(\mathrm{i}=1 \sim 6)$, the heaving, torsional and horizontal 3DOF differential equations are expressed as follows (Scanlan and Tomko, 1971);

$$
\begin{aligned}
& m \ddot{\eta}+C_{\eta} \dot{\eta}+k_{\eta} \eta=1 / 2 \rho V^{2}(2 b)\left[k H_{1}^{*} \dot{\eta} / V+k H_{2}^{*} b \dot{\phi} / V\right. \\
& \left.+k^{2} H_{3}^{*} \phi+k^{2} H_{4}^{*} \eta / b+k H_{5}^{*} \dot{\xi} / V\right] \\
& I \ddot{\phi}+C_{\phi} \dot{\phi}+k_{\phi} \phi=1 / 2 \rho V^{2}\left(2 b^{2}\right)\left[k A_{1}^{*} \dot{\eta} / V+k A_{2}^{*} b \dot{\phi} / V\right. \\
& \left.+k^{2} A_{3}^{*} \phi+k^{2} A_{4}^{*} \eta / b+k A_{5}^{*} \dot{\xi} / V\right] \\
& m \ddot{\xi}+C_{\xi} \dot{\xi}+k_{\xi} \xi=1 / 2 \rho V^{2}(2 b)\left[k P_{1}^{*} \dot{\xi} / V+k P_{2}^{*} b \dot{\phi} / V\right. \\
& \left.+k^{2} P_{3}^{*} \phi+k P_{5}^{*} \dot{\eta} / V+k^{2} P_{6}^{*} \eta / b\right]
\end{aligned}
$$

Where $\eta, \phi$ and $\xi$ are the heaving, torsional, and horizontal displacements, $m$ and $I$ are the mass and mass inertia per unit length, $C_{\eta}, C_{\phi}$ and $C_{\xi}$ are the damping coefficients, $k_{\eta}$, 
$k_{\phi}$ and $k_{\xi}$ are stiffness, $\rho$ is the air density, $b$ is the half chord length, $k$ is the reduced frequency $\left(=b \omega_{F} / V\right), \omega_{F}$ is the flutter circular frequency and $V$ is the wind velocity.

To solve the 3DOF equations, 2DOF step-by-step analysis (SBSA) (Matsumoto et al, 1995), which has many advantages (Matsumoto et al, 2007), should be expanded to 3DOF. Then the authors propose the 3DOF SBSA method and results of this method show complete agreement with those of 3DOF complex-eigenvalue analysis, even though they have different branch definitions. As an example, TB (in general, flutter onset mode) of the 3DOF SBSA method is conducted as follows. Firstly, in the torsional system, torsional motion $\left(\phi=\phi_{0} \mathrm{e}^{\lambda t}, \lambda=-\zeta_{F} \omega_{F}+i \omega_{F}\right)$ is assumed in Step 1. Secondly, in the heaving and horizontal system, both motions are generated by torsional motion, as forced vibration in Step 2. Then, by solving the simultaneous equations of the heaving and horizontal system, the amplitude ratio $\square \eta_{0} / \phi_{0}, \xi_{0} / \phi_{0}$ and the phase difference $\Psi_{\eta \phi}, \Psi_{\xi \phi}$ are calculated from this Step 2. As Step 3, in the torsional system, torsional motion is also characterized by heaving and horizontal motion, which has a certain amplitude ratio and a certain phase difference, as free vibration. From this step, the flutter damping and the flutter frequency are calculated from Eq. (28) and (29). Then, convergence calculation between the calculated flutter frequency and damping in Step 3 and the assumed flutter frequency and damping in Step 1 is carried out.

$$
\begin{aligned}
\zeta_{F}= & {\left[2 \zeta_{\phi 0} \omega_{\phi 0}+\left(\rho b^{3} / I\right) \omega_{F}\left\{-b A_{2}^{*}+A_{4}^{*}\left(\eta_{0} / \phi_{0}\right) \sin \Psi_{\eta \phi}\right.\right.} \\
& -A_{1}^{*}\left(\eta_{0} / \phi_{0}\right)\left(\zeta_{F} \sin \Psi_{\eta \phi}+\cos \Psi_{\eta \phi}\right) \\
& \left.\left.-A_{5}^{*}\left(\xi_{0} / \phi_{0}\right)\left(\zeta_{F} \sin \Psi_{\xi \phi}+\cos \Psi_{\xi \phi}\right)\right\}\right] / 2 \omega_{F} \\
\omega_{F}= & {\left[\omega_{\phi 0}^{2}+\left(\rho b^{3} / I\right) \omega_{F}^{2}\left\{-b A_{3}^{*}\right.\right.} \\
& -A_{4}^{*}\left(\eta_{0} / \phi_{0}\right)\left(\cos \Psi_{\eta \phi}-\zeta_{F} \sin \Psi_{\eta \phi}\right) \\
& -A_{1}^{*}\left(\eta_{0} / \phi_{0}\right)\left(\zeta_{F}^{2}+1\right) \sin \Psi_{\eta \phi} \\
& \left.\left.-A_{5}^{*}\left(\xi_{0} / \phi_{0}\right)\left(\zeta_{F}^{2}+1\right) \sin \Psi_{\xi \phi}\right\}\right]^{1 / 2}
\end{aligned}
$$


Similarly, in HB and horizontal branch, the flutter damping and the flutter frequency can be derived by linear summation of each aerodynamic derivative. Therefore, the effects of various aerodynamic derivatives can be clarified.

As described before, previous studies pointed out the definite role of 3DOF on coupled flutter. However, looking at the video film, when flutter occurs at prototype wind velocity of $93[\mathrm{~m} / \mathrm{s}]$, a flutter mode which looks like heaving and torsional 2DOF vibrating mode is observed. In this flutter mode, the torsional mode is predominant and the torsional fundamental mode $\mathrm{T}_{0}$ (phase difference $\Psi_{\eta \psi}=0$ [deg.]) plays an important and major role for flutter excitation at flutter onset (Matsumoto et al, 2008). Furthermore, as shown in Fig. 9, there are almost the same results or significantly small differences between the results obtained by 2DOF and 3DOF two-dimensional flutter analyses by using flutter derivatives of the Akashi Strait Bridge girder (see Fig. 8).

Looking at the static displacement characteristics as shown in Fig.10 (a), (b), when the wind velocity increases, static horizontal displacement $\xi_{\mathrm{s}}(V)$ gradually increases, and static torsional displacement $\phi_{s}(V)$ is simultaneously induced by this horizontal displacement $\xi_{\mathrm{s}}(V)$. This horizontal displacement $\xi_{\mathrm{s}}(V)$ is caused by drag force Drag. Therefore, static torsional displacement $\phi_{s}(V)$ due to horizontal displacement $\xi_{\mathrm{s}}(V)$ can be thought to be linearly related to drag force Drag as shown in Fig. 10(c). In this figure, drag force Drag is calculated from Eq. (28) by using the drag force coefficient $C_{D}$. This $\operatorname{Drag}_{-} \phi_{s}(V)$ characteristic should be a substantially structural feature as structural coupling between horizontal displacement $\xi$, and torsional displacement $\phi$ of this AFM, only influenced by drag force.

$$
\operatorname{Drag}=1 / 2 \rho V^{2} D C_{D}
$$


By the influence of these structural coupling characteristics, additional torsional displacement $\phi^{*}$ is generated by the change of drag force. While torsional response $\phi$ surely changes the drag force from the quasi-steady base, therefore, the additional pitching moment term $M^{*}\left(\phi^{*}\right)$ induced by the additional torsional displacement $\phi^{*}$ should be added in the torsional differential equation as follows;

$$
\begin{aligned}
m \ddot{\eta}+ & C_{\eta} \dot{\eta}+k_{\eta} \eta=1 / 2 \rho V^{2}(2 b)\left[k H_{1}^{*} \dot{\eta} / V\right. \\
& \left.+k H_{2}{ }^{*} b \dot{\phi} / V+k^{2} H_{3}{ }^{*} \phi+k^{2} H_{4}{ }^{*} \eta / b\right] \\
I \ddot{\phi}+ & C_{\phi} \dot{\phi}+k_{\phi} \phi=1 / 2 \rho V^{2}\left(2 b^{2}\right)\left[k A_{1}^{*} \dot{\eta} / V\right. \\
& \left.+k A_{2}{ }^{*} b \dot{\phi} / V+k^{2} A_{3}{ }^{*} \phi+k^{2} A_{4}{ }^{*} \eta / b\right]+M^{*}\left(\phi^{*}\right)
\end{aligned}
$$

When torsional displacement is $\phi$, variation of drag force $\operatorname{Drag}(\phi)$ is described by Eq.(32) from quasi-steady state.

$$
\operatorname{Drag}(\phi)=1 / 2 \rho V^{2} D^{d C_{D}} / d \alpha \phi
$$

Taking account of the relationship diagram between drag force Drag and torsional displacement $\phi_{s}(V)$, the additional torsional displacement $\phi^{*}$ caused by structural coupling is calculated by the following.

$$
\phi^{*}=-0.0148 \times \operatorname{Drag}(\phi)
$$

Where the coefficient $(-0.0148)$ is obtained from the structural property as shown in Fig.10 (c).

Finally, the additional pitching moment term $M^{*}\left(\phi^{*}\right)$ is considered as the elastic force.

$$
M^{*}\left(\phi^{*}\right)=k_{\phi} \phi^{*}=-0.0074 \times \rho V^{2} D^{d C_{D}} / d \alpha \omega_{\phi 0}^{2} \phi
$$


As shown in Eq. (31) and (34), the additional torsional displacement $\square \phi^{*}$ has the effect of decreasing torsional rigidity. Then, by using the aerodynamic derivatives of the Akashi Strait Bridge girder at angle of attack $=0$ [deg.] or -6 [deg.], 2DOF SBSA is conducted. The numerical results for the velocity-damping $(V-\delta)$ diagram and velocity-frequency $(V-\omega)$ diagram obtained by Eq. (31) are compared with the test results (Matsumoto et al, 2007) in Fig. 11 (a), (b). As shown, both results show good agreement. In particular, at near and after flutter onset, the rapid decreasing characteristic of damping as velocity increases can be well calculated by using Eq. (31). In conclusion, the authors would like to emphasize that the coupled flutter of the AFM should not be 3DOF coupled flutter from the aerodynamic point of view, but aerodynamically 2DOF coupled flutter strongly affected by the structural coupling feature between horizontal displacement and torsional response. In this study, only the first symmetric torsional and heaving vibration modes are considered. As future work, flutter analysis should be conducted taking into account the higher vibration modes and the variation of the static pitching angle along the span axis.

\section{Conclusion}

By using fundamental flutter modes, flutter modes can be resolved into torsional mode $\mathrm{T}_{0}$ or $\mathrm{T}_{180}$ and heaving mode $\mathrm{H}_{-90}$ or $\mathrm{H}_{90}$. For thin plates, from the point of view of the self excited term, the $\mathrm{T}_{0}$ mode and $\mathrm{H}_{-90}$ mode play major roles in $\mathrm{TB}$ and $\mathrm{HB}$, respectively. For flutter onset in $\mathrm{TB}, \mathrm{T}_{0}$ mode is the essential mode, on the other hand, branch switch might be encouraged mainly by the $\mathrm{H}_{-90}$ mode. Based on the $\mathrm{TB}$ characteristics flutter onset, a formula significantly similar to Selberg's formula can be 
provided. Finally, three different torsional divergence velocities are shown by using SBSA. The coupled flutter of a scale model of the Akashi-Strait Bridge can be characterized by aerodynamically conventional $2 \mathrm{DOF}(\eta, \phi)$ coupling and structural coupling between horizontal displacement and torsional motion. The simplified analytical model developed in this study explains the flutter characteristic obtained by wind tunnel tests fairly well. The authors understand that coupled flutter of plate-like structures including truss-stiffened bridge girders, flat box bridge girders and so on, would be mostly characterized by $2 \mathrm{DOF}(\eta, \phi)$ coupling. Of course, particular sections in which aerodynamic derivatives associated with horizontal motion are comparatively larger than those associated with heaving and torsional motion would show 3DOF coupled flutter.

Finally, the authors would like to acknowledge Mr. Y. Kato of Kyoto University for his contribution on related wind tunnel tests in this research and Mr. S. Kusuhara of Honshu-Shikoku Bridge Expressway Company Limited for giving experimental data on the AFM.

\section{References}

M. Matsumoto, Y. Nihara, Y. Kobayashi, H. Sato, H. Hamasaki: "Flutter mechanism and its Stabilization of Bluff Bodies”, Proc. of $9^{\text {th }}$ ICWE, pp 827-838, 1995.

M. Matsumoto, K. Okubo, Y. Ito, H. Matsumiya, G. Kim: "Branch characteristics of coupled flutter and its stabilization" Proc. of 12 $2^{\text {th }}$ ICWE, Vol. 2, pp 2240-2246, 2007. 
A. Selberg: "Oscillation and aerodynamic stability of suspension bridges". ACTA Polytechnica Scandinavica, Civil Engineering and Construction Series 13, 1961.

T. Miyata, K. Tada, H. Sato, H. Katsuchi, Y. Hikami: "New Findings of Coupled-Flutter in Full Model Wind Tunnel Tests of the Akashi Kaikyo Bridge”, Proc. of Symp. on Cable-Stayed and Suspension Bridges, pp 163-170, 1994.

H. Sato, M. Kitagawa, T. Kanazaki, R. Toriumi, H. Katsuchi: "Wind Tunnel Test of the Akashi Kaikyo Bridge using Full Bridge Model", Journal of Wind Engineering No. 68, pp 25-36, 1996.

Scanlan, R.H. and Tomko, J.J.: "Airfoil and Bridge Deck Flutter Derivatives”, Journal of Eng. Mech. Division, 97, EM6, American Society of Civil Engineering, pp 1717-1737, 1971.

M. Matsumoto, H. Matsumiya, S. Fujiwara, Y. Ito: "On flutter fundamental modes, reduction of similar Selberg's formula and torsional divergence instability basing on SBSA" $6^{\text {th }} B B A A, 2008$. 